\title{
Hydrolysis profiles of formalin fixed paraffin-embedded tumors based on IOD (integrated optical density) and nuclear texture feature measurements
}

\author{
Margareta Fležar ${ }^{\mathrm{a}, *}$, Marija Us-Krašovec ${ }^{\mathrm{a}}$, \\ Mario Žganec ${ }^{b}$, Jaka Lavrenčak ${ }^{a}$ and \\ Rastko Golouh ${ }^{\text {a }}$

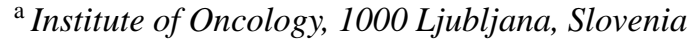 \\ ${ }^{\mathrm{b}}$ Faculty of Electrical Engeneering, 1000 Ljubljana, \\ Slovenia
}

Received 11 August 1999

Accepted 31 January 2000

The aim of the study was to determine optimal hydrolysis time for the Feulgen DNA staining of archival formalin fixed paraffin-embedded surgical samples, prepared as single cell suspensions for image cytometric measurements.

The nuclear texture features along with the IOD (integrated optical density) of the tumor nuclei were analysed by an automated high resolution image cytometer as a function of duration of hydrolysis treatment (in $5 \mathrm{~N} \mathrm{HCl}$ at room temperature). Tissue blocks of breast carcinoma, ovarian serous carcinoma, ovarian serous tumor of borderline malignancy and leiomyosarcoma were included in the study.

IOD hydrolysis profiles showed plateau between 30 and $60 \mathrm{~min}$ in the breast carcinoma and leiomyosarcoma, and between 40 and $60 \mathrm{~min}$ in the ovarian serous carcinoma and ovarian serous tumor of borderline malignancy. Most of the nuclear texture features remained stable after $20 \mathrm{~min}$ of hydrolysis treatment.

Our results indicate that the optimal hydrolysis time for IOD and for nuclear texture feature measurements, was between 40 and $60 \mathrm{~min}$ in the cell preparations from tissue blocks of three epithelial and one soft tissue tumor.

Keywords: Image cytometry, formalin fixed paraffinembedded tumors, Feulgen reaction, hydrolysis, nuclear texture features

\footnotetext{
*Correspondence to: Margareta Fležar, MD, Dept. Cytopathology, Institute of Oncology, Zaloška 2, 1105 Ljubljana, Slovenia. Tel.: +386 6113220 99; Fax: +386 611314 180; E-mail: mflezar@onkoi.si.
}

\section{Introduction}

Fresh material is considered technically superior to archival one for the cytometric measurements; nevertheless the archival surgical samples with accompanying follow-up data of the patients are a valuable source of research material in retrospective studies. The formalin fixed and paraffin embedded archival tissue samples processed according to Hedley's method yield suspensions of single cells that can be used for flow cytometric DNA ploidy measurements $[11,12]$. If the cell suspensions are prepared as monolayers of separate cells on glass slides, they can be used for image cytometric DNA ploidy and nuclear texture measurements as well [10,21].

The majority of the steps in the preparation of single cell suspensions from paraffin embedded tissue which affect the chromatin, such as the steps of enzymatic dissociation, are standardised. The optimal time of acid hydrolysis in $5 \mathrm{~N} \mathrm{HCl}$, required before Feulgen staining, should be defined in every study for different tissue types and fixations [10]. Commonly, the optimal hydrolysis time is determined from the integrated optical density (IOD) profile of the study sample. However, there is no substantial information on the effect of hydrolysis treatment on nuclear texture features in the cell suspensions from formalin fixed paraffinembedded tissues. In our previous study on fresh cytologic material, we have shown that the plateau of hydrolysis curve, which represents the optimal time for hydrolysis treatment, was shorter, if nuclear texture feature values instead of IOD values were plotted on $x$-axis [7].

The aim of the present study was to determine the optimal hydrolysis times of tumors, represented by the cell preparations from archival biopsy samples, in order to achieve optimal Feulgen staining for image 
cytometric measurements. For this purpose, hydrolysis profiles of the breast carcinoma, ovarian serous carcinoma, ovarian serous tumor of borderline malignancy and leiomyosarcoma were established from IOD measurements. Furthermore, the hydrolysis profiles of these tumors were determined from nuclear texture feature measurements. Diploid cell populations of tumors, as well as aneuploid ones, where present, were specifically analysed.

\section{Materials and methods}

Three different categories of epithelial tumors and one soft tissue tumor were included in the study. The epithelial tumor category was represented by breast carcinomas (3 cases), ovarian serous adenocarcinomas ( 3 cases) and serous ovarian tumors of borderline malignancy ( 3 cases). The soft tissue tumor category was represented by three different leiomyosarcomas. The samples were derived from surgical resection specimens, that were originally fixed in buffered $4 \%$ formaldehyde and paraffin embedded. All the specimens were collected between 1990 and 1994.

Seven $50 \mu \mathrm{m}$ sections, followed by one $5 \mu \mathrm{m}$ thick section, were cut from each tumor. The thin section was stained with hematoxylin-eosin (HE) to check for the presence of the tumor, while cell suspensions were prepared from the thick sections according to modified Hedley method $[11,12]$. The cell suspensions were produced by using $0.1 \%$ protease incubated at room temperature with magnetic stirring for $45 \mathrm{~min}$.

Three filter slides for each of the eight hydrolysis times were prepared from the cell suspensions of each tumor. All slides were air-dried. Afterwards, they were fixed in Böhm-Sprenger fixative for 30 to 120 min. One additional filter slide was prepared from each tumor cell suspension and stained according to the Giemsa method to check the morphology and preservation of the cells.

Hydrolysis was performed in $5 \mathrm{~N} \mathrm{HCl}$ at room temperature for $10,20,30,40,50,60,75$ and $90 \mathrm{~min}$. The slides were stained according to modified Feulgen staining with thionin (thionin acetate, colour index 52000, Lot No. MY 00209 TW, Aldrich Chemical Company, Inc., Milwaukee, USA) for $60 \mathrm{~min}$ [6].

Image analysis of cell samples was performed with an automated, high resolution image cytometer (CytoSavant $^{\mathrm{TM}}$, Oncometrics Imaging Corp., Vancouver, BC, Canada). The images of the nuclei are projected onto a $1320 \times 1035$ pixel scientific CCD positioned in the primary image plane of a $20 \times$ Plan-Apochromatic objective lens (NA 0.75). The individual sensing elements of the CCD are $6.8 \mu \mathrm{m} \times 6.8 \mu \mathrm{m}$ squares and sense light on their entire surface (100\% fill factor). A $600 \pm 10 \mathrm{~nm}$ filter is used because it corresponds to the spectral region of peak absorption of the nuclear stain. Standard shading correction and correction for the local backgroud are incorporated in the system. The hardware and its performance are described in detail in the sources listed in the references $[8,13]$.

Nuclear images were acquired automatically by random sampling by means of the data acquisition program Acquire, incorporated into the system. All nuclear images were acquired in the best focus and the edge relocation algorithm was applied for precise segmentation of nuclei from the background [17]. The representativity and the focus of all the nuclei acquired was checked. Afterwards, all the artifacts, damaged nuclei or nuclei in poor focus were excluded from the analysis.

Over 100 nuclear features were calculated from each of the nuclear images. The nuclear features included common morphometric features (area, diameter, shape features, etc.), descriptive statistics of chromatin distribution (integrated optical density (IOD), variance of optical density (OD), OD skewness and kurtosis), discrete chromatin distribution (area and shape of high, medium, low density chromatin components, average distance between chromatin components of the same optical densities, etc.), Markovian texture features (entropy, energy, correlation, homogeneity, cluster shade, etc.), local extreme features (number of local minima and maxima in the image) and run length texture features (short run emphasis, long run emphasis, grey level nonuniformity, etc.). Exact formulas and feature descriptions can be found in the references [5].

The nuclear features of the cell population in $\mathrm{G}_{0} \mathrm{G}_{1}$ peak of DNA histogram in the diploid tumors were included in the statistical analyses. In the aneuploid tumors (all breast carcinomas and two ovarian serous carcinomas), the cells belonging to the $\mathrm{G}_{0} \mathrm{G}_{1}$ aneuploid peak were also analysed separately. In two leiomyosarcomas, less than 100 nuclear images were acquired per slide, therefore they were excluded from further analysis. Each study group contained between 223 and 1402 objects (nuclei).

The hydrolysis profiles were established from the IOD and nuclear texture feature measurements for all tumor types. Statistical significance of any differences in the nuclear features values of the cells from one tumor, but with different duration of hydrolysis treat- 
(A)
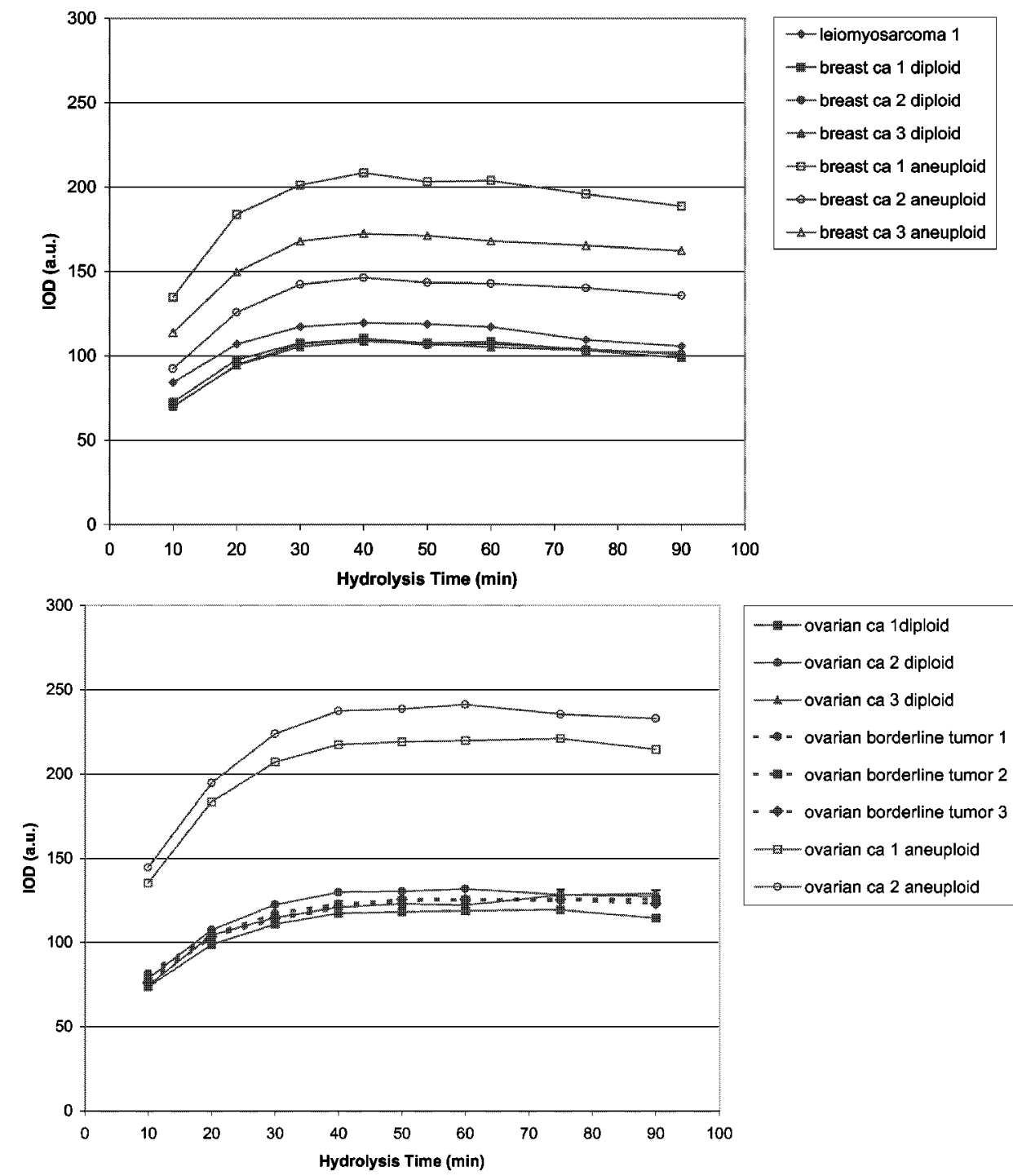

Fig. 1. (A) Hydrolysis profiles of diploid and aneuploid cell populations in breast carcinoma, and of diploid cell population in leiomyosarcoma based on IOD (integrated optical density). (B) Hydrolysis profiles of diploid and aneuploid tumor populations in ovarian serous carcinoma and of diploid cell populations in ovarian serous tumor of borderline malignancy based on IOD.

ment, were tested with ANOVA (Tukey Studentized rang test). The differences were considered statistically significant at $p<0.05$.

\section{Results}

\subsection{Hydrolysis profiles established from IOD measurements}

In the hydrolysis profiles of the breast carcinomas and leiomyosarcoma, IOD was increasing from 10 to
30 min, while in the ovarian serous tumors, IOD was increasing from 10 to $40 \mathrm{~min}$ of hydrolysis. Thereafter, the IOD values reached a plateau in all the tumors and IOD remained relatively constant for 30-60 min. Afterward, the IOD values slightly decreased with further hydrolysis (Fig. 1(A, B)). The diploid and the aneuploid cell populations of the same tumors, namely breast carcinoma and ovarian serous carcinoma, had similar IOD hydrolysis profiles (Fig. 1(A, B)). The IOD values of aneuploid cells were higher already after $10 \mathrm{~min}$ of hydrolysis treatment and remained higher thereafter. 


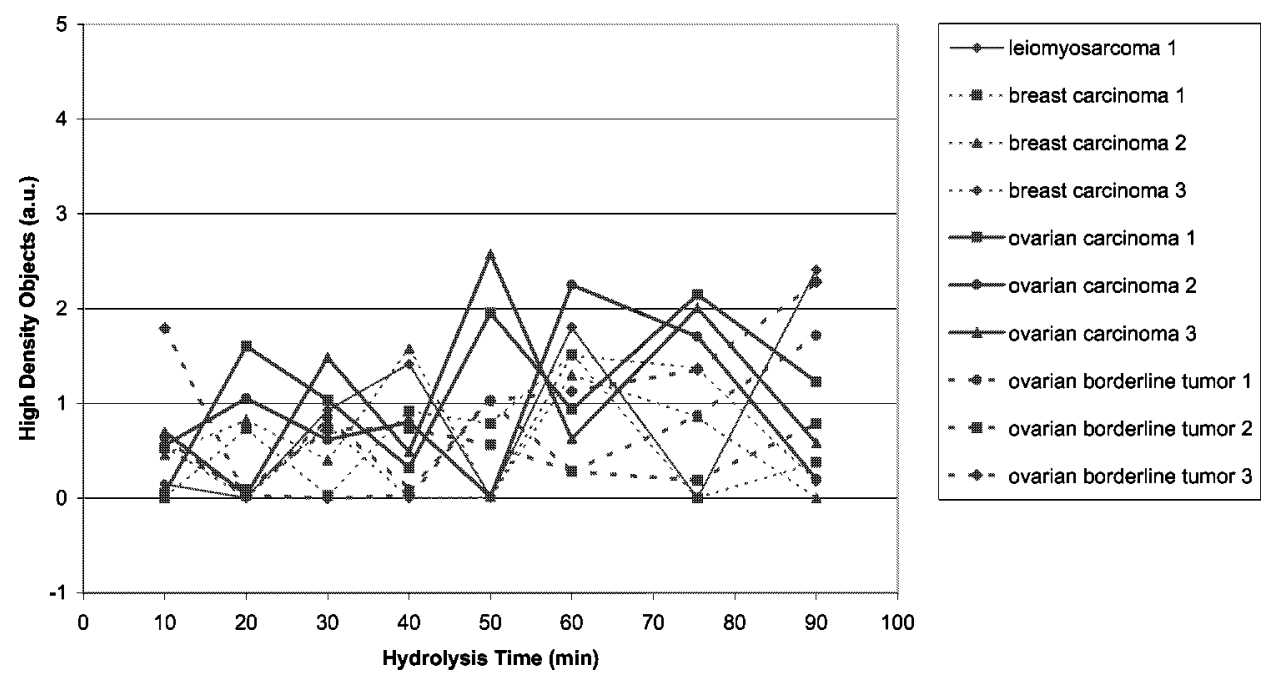

Fig. 2. Hydrolysis profiles based on "high density objects" in breast carcinoma, ovarian serous adenocarcinoma, ovarian serous tumor of borderline malignancy and leiomyosarcoma.

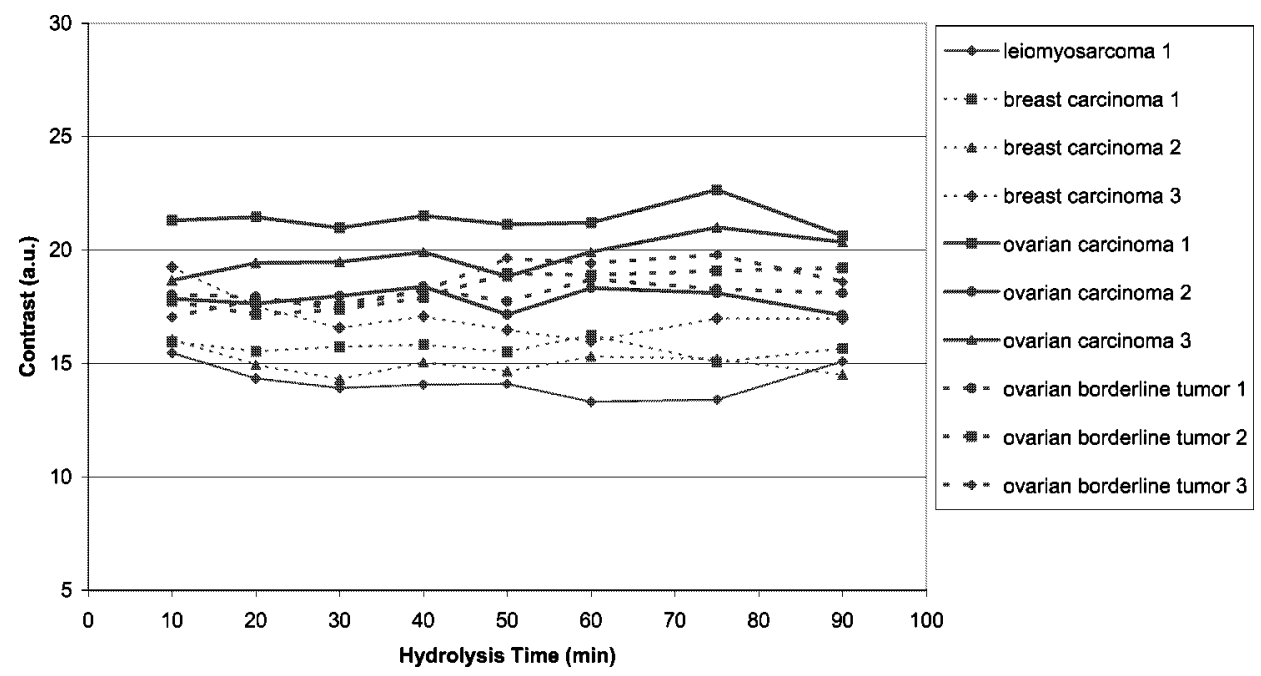

Fig. 3. Hydrolysis profiles based on "contrast" in breast carcinoma, ovarian serous adenocarcinoma, ovarian serous tumor of borderline malignancy and leiomyosarcoma.

\subsection{Hydrolysis profiles established from nuclear texture feature measurements}

The hydrolysis profiles nuclear texture features established from nuclear texture feature measurements showed relatively constant values of the majority of nuclear texture features as a function of hydrolysis time. However, some nuclear texture features reached constant values after 20 or $30 \mathrm{~min}$ of hydrolysis treatment. In the aneuploid tumors with diploid $\mathrm{G}_{0} \mathrm{G}_{1}$ cell population, the nuclear texture features hydrolysis profiles were similar for both, the diploid and aneuploid cell populations.
The most variable values between different hydrolysis times were found in the group of discrete nuclear texture features for all tumors, however, the differences were not statistically significant. The hydrolysis profile of "high density objects", which measures the number of high density clumps in the nucleus, is also representative for other features of this group (not shown here) (Fig. 2).

In the group of Markovian texture features, the "contrast" is based on the estimation of intensity difference between neighbouring pixels, yielding increasing values with the increasing number and magnitude of 
(A)

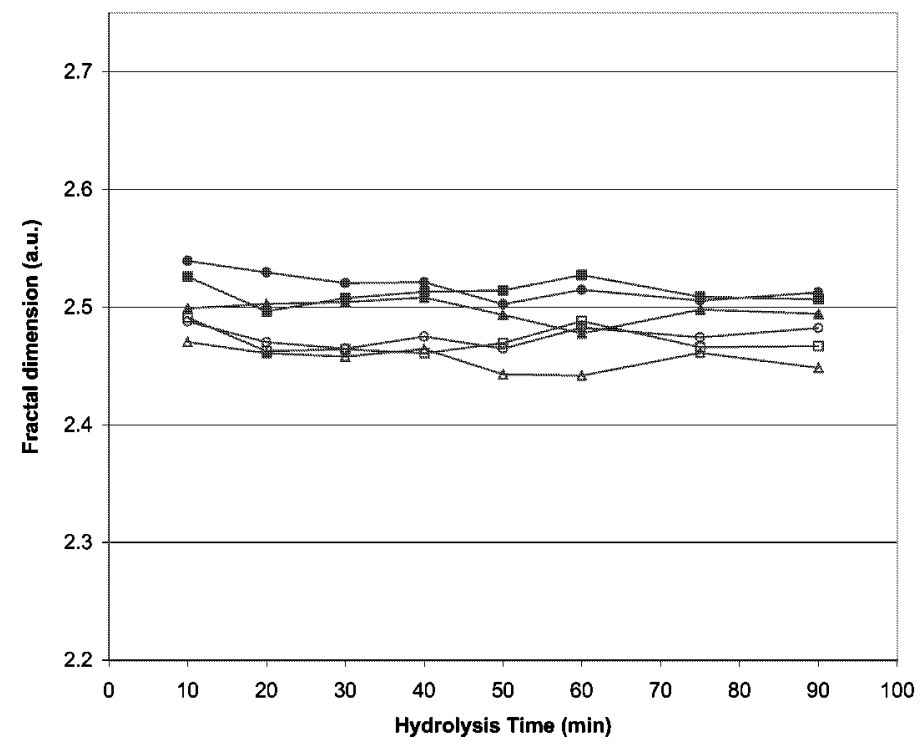

$\rightarrow$ breast ca 1 diploid

- breast ca 2 diploid

- breast ca 3 diploid

- breast ca 1 aneuploid

-breast ca 2 aneuploid

- breast ca 3 aneuploid

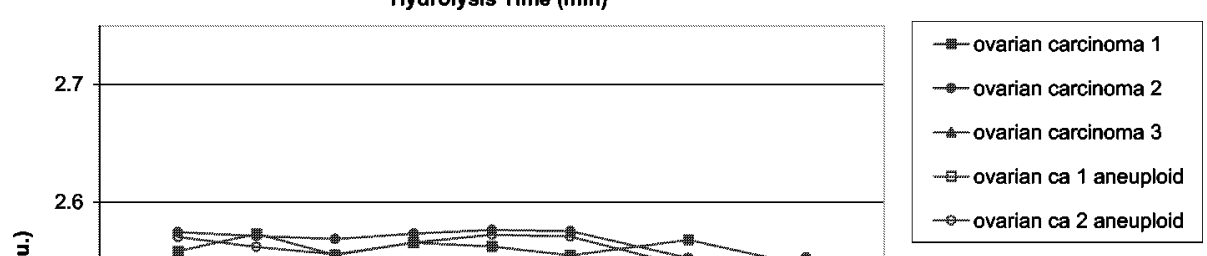

(B)

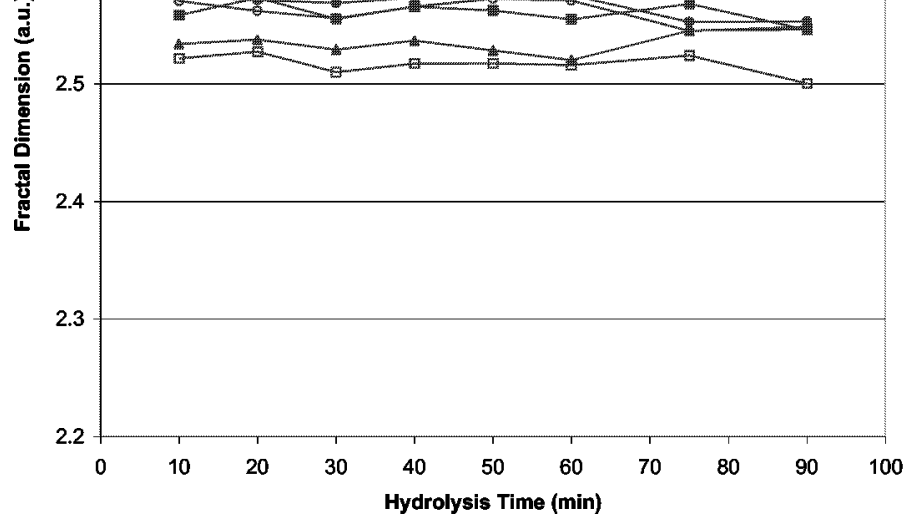

Fig. 4. (A) Hydrolysis profiles based on "fractal dimension" in diploid and aneuploid cell populations of breast carcinoma. (B) Hydrolysis profiles based on "fractal dimension" in diploid and aneuploid cell populations of ovarian serous adenocarcinoma.

density variation between chromatin particles. The values of this feature did not change significantly after 20 min of hydrolysis treatment in the breast carcinomas (Fig. 3). In other tumors, the "contrast" did not change significantly throughout the hydrolysis, except in two ovarian serous tumors of borderline malignancy after 50 and 75 min of hydrolysis, respectively. The results of the analysis for other features of this group were similar.

In the fractal texture features group, the "fractal dimension" yields high values to the nuclear images with spatially frequent, high contrast variations in chro- matin condensation. In the breast carcinoma, we found no significant differences between 20 and $60 \mathrm{~min}$ of hydrolysis (Fig. 4A). In one of the three ovarian serous carcinomas (in ovarian serous carcinoma 2) we found significant differences in fractal dimension only after $75 \mathrm{~min}$ of hydrolysis treatment (Fig. 4B). The values of this feature were constant for two serous ovarian tumors of borderline malignancy as a function of the hydrolysis time, in one tumor the values were stable only after $30 \mathrm{~min}$ of hydrolysis (Fig. 4B). Analysing other features of this group, we obtained similar results. 


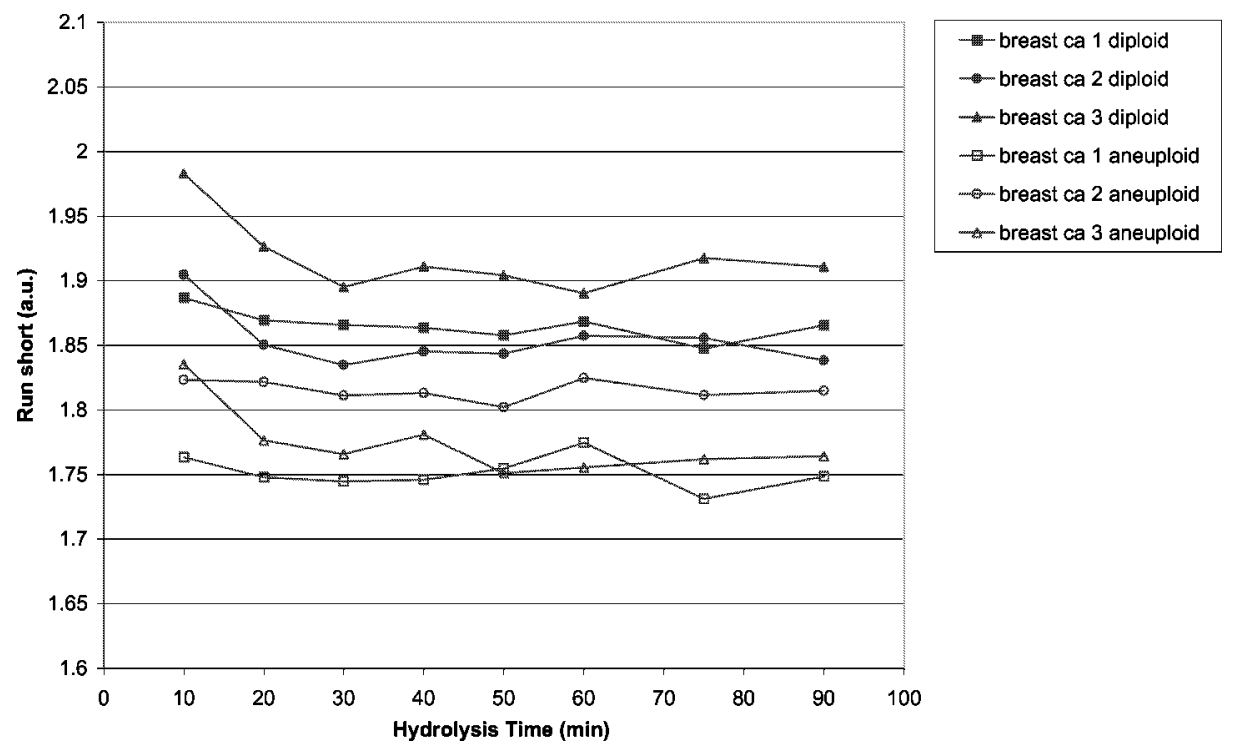

Fig. 5. Hydrolysis profiles based on "short run emphasis" in diploid and aneuploid cell populations of breast carcinoma.

In the run length feature group, the "short run emphasis" gives maximal values to the nuclei with small chromatin clumps of different condensation. The feature values were invariable as a function of hydrolysis time in the majority of the tumors. The exception were two breast carcinomas (breast carcinomas 2 and 3 ) where the values did not change significantly after 20 and 30 min of hydrolysis (Fig. 5).

\section{Discussion}

It has been well established that both fixation and hydrolysis time affect DNA staining and subsequently DNA amount (IOD) measurements [1-4,9,14-16,18, 20,22]. In contrast, relatively little attention has been paid to these issues in measurements of nuclear texture features $[4,9]$.

In this study, we found that the optimal hydrolysis time (hydrolysis in $5 \mathrm{~N} \mathrm{HCl}$ at room temperature) for cell suspensions from formalin fixed paraffinembedded tumors is between 30 and $60 \mathrm{~min}$ for the breast carcinoma and leiomyosarcoma, and between 40 and 60 min for the ovarian serous tumors.

Our experiments show that the plateau on the hydrolysis curve established from IOD measurements is between 30 and $60 \mathrm{~min}$ in the breast carcinoma and leiomyosarcoma, and between 40 and $60 \mathrm{~min}$ in the ovarian serous carcinoma and ovarian serous tumor of borderline malignancy (Fig. 1).
We additionally defined the optimal hydrolysis time by nuclear texture feature analysis, since nuclear texture features have been proposed to yield additional (and possibly superior) information compared to DNA ploidy, and may be of both diagnostic and prognostic significance [19]. The plateau values of the nuclear texture features cover the range of the optimal hydrolysis time of IOD, since they are either constant as a function of hydrolysis time or ultimately reach the plateau after 20 or $30 \mathrm{~min}$ of hydrolysis treatment (Figs 2-5). The exception are discrete texture features, i.e., high density objects, where the values are quite diverse between different hydrolysis times. Discrete texture features, namely high, medium and low density objects, are expressed as integer values in contrast to other features, which are real numbers. This fact as well as the way these features are calculated makes them sensitive to small changes in nuclear image, which leads to unstable feature values and their high CV's. This limits the use of such features to the cases, where feature values can be averaged over a very large number of cell nuclei.

The established optimal hydrolysis time for the cell preparations from formalin fixed paraffin-embedded tumors in our study confirms the range 40-60 min reported earlier for similar preparations from hyperplastic laryngeal epithelium [23]. Moreover, the optimal hydrolysis time for DNA staining in our study is supported by the constant values of nuclear texture features in this range. 
The IOD and specifically nuclear texture features in the cell suspensions from formalin fixed paraffinembedded tissues in our study reach the plateau on hydrolysis curve earlier than cells from fresh tissues fixed mainly by alcohol fixatives [7,22]. It is known that formaldehyde fixation induces the formation of cross-links between DNA and nucleoproteins, which enhances the access of dye to potential dye-binding sites [14].In contrast, previous studies on the effect of formalin fixation on IOD values, where the IOD plateau in the formalin fixed cells appeared later than in the alcohol fixed cells, analysed the cells obtained from fresh tissue by imprinting, or from cell cultures, where no enzymatic disaggregation was used in the preparation procedure. We suppose that the DNA of cells obtained from formalin fixed paraffin-embedded tissues is more accessible to dyes after protease digestion. However, there is no clear evidence in what manner the protease digestion step affects the nuclear membrane or nuclear proteins themselves.

In addition, the plateau range of nuclear texture features in the cell preparations from formalin fixed paraffin-embedded tissue is not restricted only to the IOD plateau. Furthermore, the plateau of the nuclear texture feature in such cells is even longer than that of IOD (Figs 2-5). The cross-linking of nuclear proteins to DNA by formalin fixation forms a mesh, which probably entraps depolymerized DNA fragments within chromatin, and delays their extraction with longer hydrolysis times [14]. The longer plateau probably reflects that the chromatin structure is preserved better and longer in formalin fixed tissue during hydrolysis treatment. In contrast, alcohol fixed tissue is less protected against the harmful effect of hydrolysis and depolymerized DNA fragments are extracted earlier. Consequently, IOD decreases and nuclear texture features deteriorate earlier [7,22].

In our study, we found that the optimal hydrolysis times are identical for the diploid and aneuploid cell populations of the same tumor (Fig. 1(A, B)). This implies that the optimal DNA staining of different cell populations from the same tumor can be assured at the chosen optimal hydrolysis time. Besides, we observed higher IOD of the aneuploid tumor cells compared to the diploid cells of the same tumor already after 10 min of hydrolysis treatment, which probably reflects the higher DNA content available for the staining at the beginning of the procedure.

To summarize, we have shown that the optimal hydrolysis time for Feulgen based DNA staining of the cell suspensions from formalin fixed paraffin- embedded tumors, which is commonly used in image cytometry, is between 30 and $60 \mathrm{~min}$ for the breast carcinoma and leiomyosarcoma, and between 40 and 60 min for the ovarian serous carcinoma and ovarian serous tumor of borderline malignancy. The IOD based optimal hydrolysis time is supported by the constant values of nuclear texture features in this range. Also, the optimal hydrolysis times of the diploid and aneuploid cell populations from the tumors in the study fall into the above range. Considering longer plateau times of nuclear texture features, it appears that chromatin structure in the cells from formalin fixed paraffinembedded tumors is preserved longer during hydrolysis treatment.

\section{Acknowledgments}

The authors thank Mrs. Vesna Gril and Miss Marijana Matic for their technical assistance. This work was partially supported by the Slovenian Ministry of Science and Technology (grant J3-7956).

\section{References}

[1] M. Aubele, G. Burger and K. Rodenacker, Problems concerning the quality of DNA measurements on Feulgen stained imprints. A study of five fixation techniques, Analyt. Quant. Cytol. Histol. 16 (1994), 226-232.

[2] J.P.A. Baak, E. Noteboom and J.J.M. Koevoets, The influence of fixatives and other variations in tissue processing on nuclear morphometric features, Analyt. Quant. Cytol. Histol. 4 (1989), 219-224.

[3] R.L. Becker Jr., U.V. Mikel, Interrelation of formalin fixation, chromatin compactness and DNA values as measured by flow and image cytometry, Analyt. Quant. Cytol. Histol. 12 (1990), 333-341.

[4] Y. De Launoit, R. Kiss and A. Danguy, Influence of smear preparation and fixatives on the DNA ploidy and morphonuclear features of the MXT mammary tumor and normal tissues in the mouse, Cytometry 11 (1990), 691-699.

[5] A. Doudkine, C. MacAulay, N. Poulin and B. Palcic, Nuclear texture measurements in image cytometry, Pathologica 87 (1995), 286-299.

[6] P. van Duijin, A histochemical specific thionine- $\mathrm{SO}_{2}$ reagent and its use in a bicolor method for deoxyribonucleic acid and periodic acid-Schiff positive substances, J. Histochem. Cytochem. 4 (1956), 55-63.

[7] M. Fležar, A. Doudkine and M. Us-Krašovec, The effect of primary fixation with standard postfixation and the duration of hydrolysis on nuclear features in image cytometry, Anal. Cell. Pathol. 17 (1998), 131-144. 
[8] D.M. Garner, A. Harrison, C. MacAulay and B. Palcic, CytoSavant ${ }^{\mathrm{TM}}$ and its use in automated screening of cervical smears, in: Compendium on the Computerized Cytology and Histology Laboratory, Tutorials of cytology, Chicago, 1994, pp. 346-352.

[9] F. Giroud and M.P. Montmasson, Reevaluation of optimal Feulgen reaction for automated cytology. Influence of fixatives, $A n$ alyt. Quant. Cytol. Histol. 11 (1989), 87-95.

[10] G. Haroske, F. Giroud, A. Reith and A. Böcking, 1997 ESACP consensus report on diagnostic DNA image cytometry. Part I: Basic considerations and recommendations for preparation, measurement and interpretation. European Society for Analytical Cellular Pathology, Anal. Cell. Pathol. 17 (1997), 189-200.

[11] D.W. Hedley, M.L. Friedlander, I.W. Taylor, C.A. Rugg and E.A. Musgrove, Method for analysis cellular DNA content of paraffin-embedded pathological material using flow cytometry, J. Histochem. Cytochem. 31 (1983), 1333-1335.

[12] T. Heiden, N. Wang and B. Tribukait, An improved Hedley method for preparation of paraffin-embedded tissues for flow cytometric analysis of ploidy and S-phase, Cytometry 12 (1991), 614-621.

[13] B. Jaggi, S. Poon, B. Pontifex, J. Fengler and B. Palcic, A quantitative microscope for image cytometry, J. SPIE 1448 (1991), 89-97.

[14] P.T.T. Kjellstrand, Mechanisms of the Feulgen acid hydrolysis, J. Microscop. 119 (1980), 391-396.

[15] P.T.T. Kjellstrand, Temperature and acid concentration in the search for optimum Feulgen hydrolysis conditions, J. Histochem. Cytochem. 25 (1977), 129-134.
[16] V.M. Kotelnikov and L.L. Litinskaya, Comparative studies of Feulgen hydrolysis for DNA, I. Influence of different fixatives and polyethylene glycols, Histochem. 71 (1981), 145-153.

[17] C. MacAulay and B. Palcic, An edge relocation segmentation algorithm, Analyt. Quant. Cytol. Histol. 12 (1990), 165-171.

[18] U.V. Mikel and R.L. Becker Jr., A comparative study of quantitative stains for DNA in image cytometry, Analyt. Quant. Cytol. Histol. 13 (1991), 253-260.

[19] B. Palcic, Nuclear texture: Can it be used as a surrogate endpoint biomarker?, J. Cell. Biochem. 19(Suppl.) (1994), 40-46.

[20] N.M. Poulin, J.B. Matthews, K.A. Skov and B. Palcic, Effects of fixation method on cytometric measurement of DNA content and distribution in cells stained for fluorescence with propidium iodide, J. Histochem. Cytochem. 42 (1994), 1149-1156.

[21] A. Reith and H. Danielsen, Assessment of DNA ploidy in tumor material. Preparation and measurement by image cytometry, in: Compendium on the Computerized Cytology and Histology Laboratory, G.L. Wied, P.H. Bartels, D. Rosenthal and U. Schenk, eds, Tutorials of cytology, Chicago, 1994, pp. 185193.

[22] E.K.W. Schulte and D.H. Wittekind, Standardization of Feulgen reaction. The influence of chromatin condensation on the kinetics of acid hydrolysis, Analyt. Cell. Pathol. 2 (1990), 149157

[23] T.C. Stenersen, H. Danielsen, G. Farrants and A. Reith, Caveats in the use of archival material for DNA ploidy analysis by image cytometry, Analyt. Cell. Pathol. 7 (1994), 217-233. 


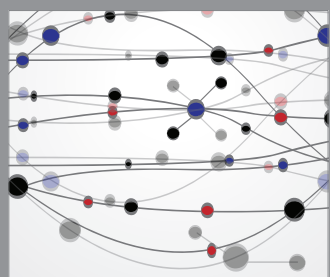

The Scientific World Journal
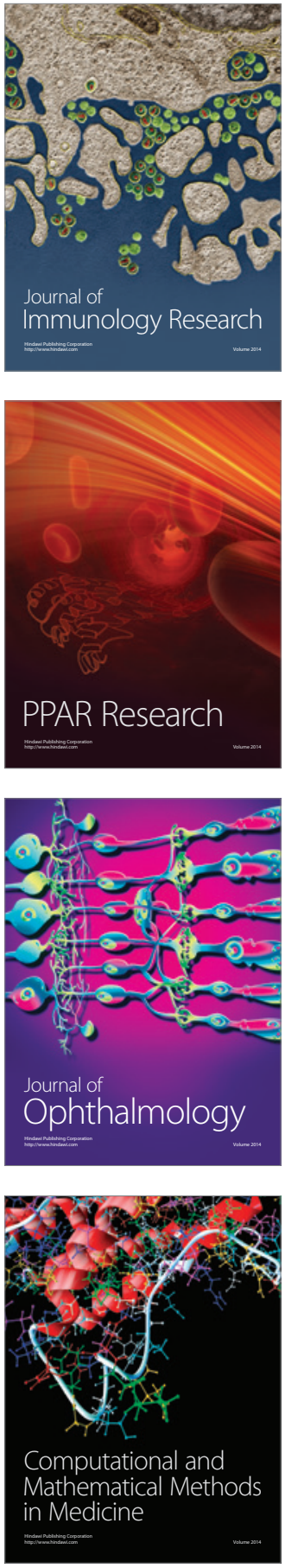

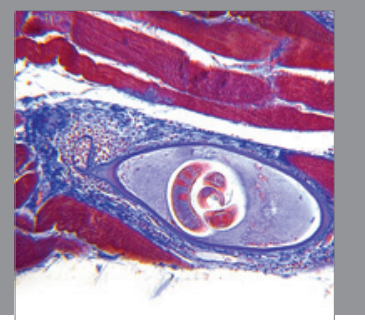

Gastroenterology

Research and Practice
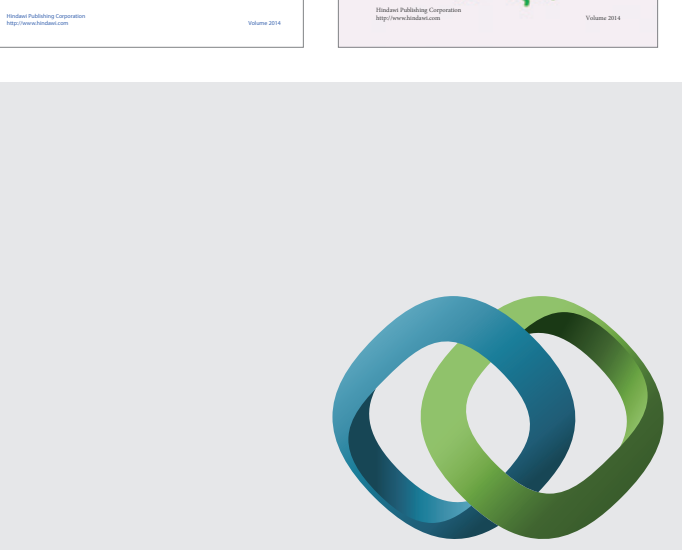

\section{Hindawi}

Submit your manuscripts at

http://www.hindawi.com
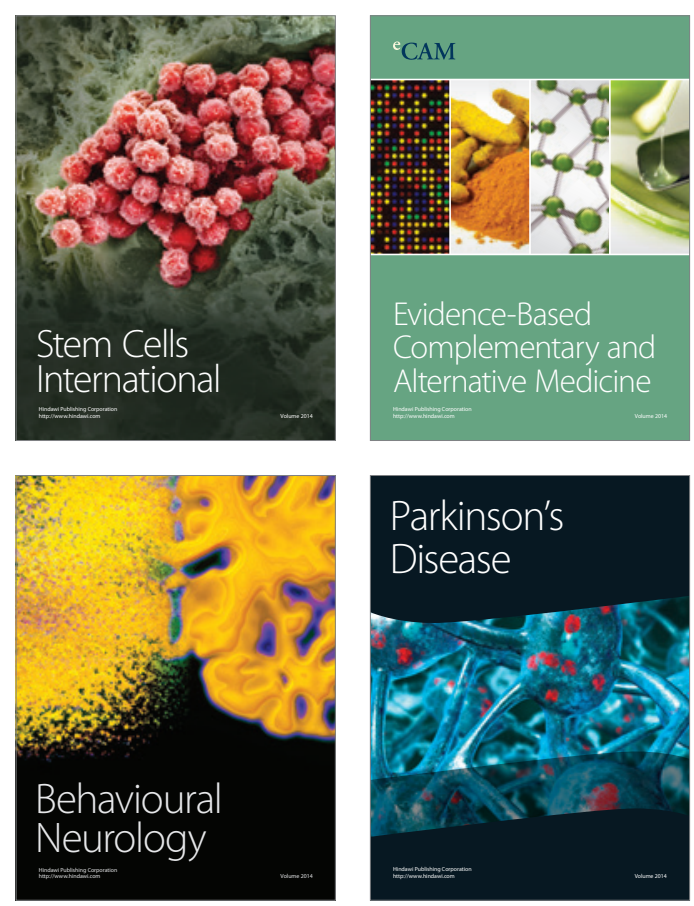

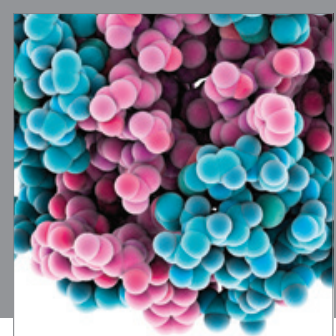

Journal of
Diabetes Research

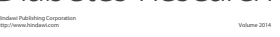

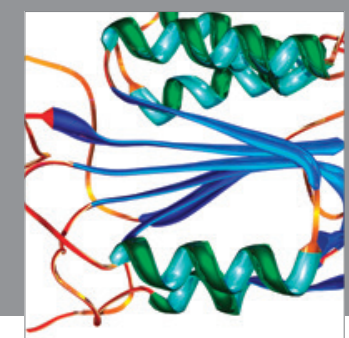

Disease Markers
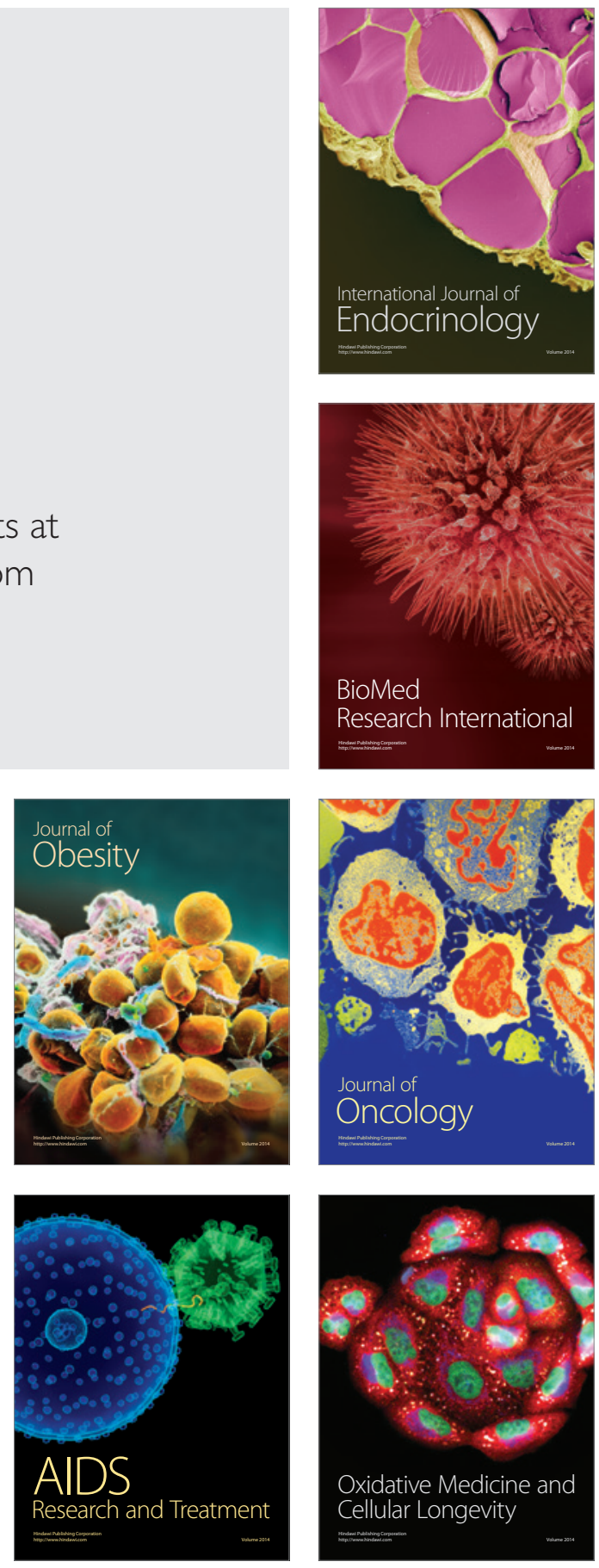